\title{
Pembrolizumab in der neoadjuvanten Behandlung des frühen triple-negativen Mammakarzinoms
}

\author{
Friederike Dierks ${ }^{1}$ Elisabeth Pietsch ${ }^{1}$ Jürgen Dunst ${ }^{2}$ \\ Online publiziert: 19. Juni 2020 \\ (c) Der/die Autor(en) 2020
}

Hintergrund Ungefähr $15 \%$ aller Mammakarzinome sind triple-negative Mammakarzinome (TNBC). Mit dieser Diagnose werden allerdings keine Tumoren mit einheitlicher Biologie beschrieben, sondern ein heterogenes Bild unterschiedlicher biologischer Subtypen, die auf konventionelle Chemotherapie verschieden ansprechen. Da beim TNBC keine bekannten Hormon- oder Wachstumsfaktorrezeptoren vermehrt exprimiert werden, die für eine gezielte Therapie genutzt werden könnten, ist eine konventionelle Chemotherapie bisher die einzige zugelassene systemische Therapie $[1,7]$. Bei frühen, nichtmetastasierten TNBC wird aktuell eine neoadjuvante anthrazyklin-/taxanbasierte Chemotherapie empfohlen [7].

Das hohe Rezidiv- und Metastasierungsrisiko des TNBC im Vergleich zu anderen Mammakarzinomsubtypen und das prognostisch ungünstige Metastasierungsmuster mit vorwiegend viszeralen und zentralnervösen Metastasen verdeutlicht die Notwendigkeit weiterer, spezifischer Therapiemöglichkeiten [1, 5]. Eine Option könnte der monoklonale PD-1-Antikörper Pembrolizumab sein. Bisherige Studien belegten die Antitumorwirksamkeit von Pembrolizumab bei frühen Formen des TNBC sowie ein akzeptables Sicherheitsprofil. Ziel der vorliegenden Studie KEYNOTE522 war es, herauszufinden, ob durch den neoadjuvanten Einsatz von Pembrolizumab zusätzlich zu einer etablierten neoadjuvanten Chemotherapie signifikant mehr pathologische Komplettremissionen (pCR) und ein längeres ereignisfreies Überleben (EFS) erreicht werden können. Die pCR gilt als Surrogatmarker für eine längere Überlebenszeit [2].

Originalpublikation Schmid P, Cortes J, Pusztai L et al (2020) Pembrolizumab for early triple-negative breast cancer. N Engl J Med 382(9):810-821. https://doi.org/10.1056/NEJMoa1910549

$\triangle$ Prof. Dr. med. Jürgen Dunst

Juergen.Dunst@uksh.de

1 Kiel, Deutschland

2 Klinik für Strahlentherapie, Universitätsklinikum Schleswig-Holstein, Campus Kiel, Feldstr. 21, 24105 Kiel, Deutschland
Patientenkollektiv und Methoden Um den möglichen Gewinn eines zusätzlichen Einsatzes von Pembrolizumab zu untersuchen, wurden in dieser randomisierten, doppelblinden Phase-3-Studie Patienten mit einem erstdiagnostizierten, unbehandelten TNBC im Stadium II oder III in eine neoadjuvante und eine adjuvante Therapiegruppe randomisiert (Ratio Pembrolizumab- zu Plazebogruppe: 2:1). Eingeschlossen wurden Patienten mit nichtmetastasiertem TNBC (cT1c und N1-2 oder cT2-4 und N0-2) ohne aktive Autoimmunerkrankungen, Immundefekte oder immunsuppressive Therapien innerhalb der letzten 2 Wochen.

Eine Gruppe $(n=784)$ erhielt 4 Zyklen Pembrolizumab (jeweils $200 \mathrm{mg}$ ) alle 3 Wochen plus Paclitaxel und Carboplatin, die andere $(n=390) 4$ Zyklen Plazebo alle 3 Wochen plus Paclitaxel und Carboplatin. Beide Gruppen erhielten 4 weitere Zyklen Pembrolizumab bzw. Plazebo plus Doxorubicin/Cyclophosphamid oder Epirubicin/Cyclophosphamid.

Im Rahmen der postoperativen Behandlung erhielten die Patienten bis zu 9 Zyklen Pembrolizumab bzw. Plazebo alle 3 Wochen. Primäre Endpunkte der Studie waren die pCR zum Zeitpunkt der Operation, definiert als ypT0/Tis ypN0 und das EFS ab dem Zeitpunkt der Randomisierung. Die Hypothese war eine Verbesserung der pCR-Rate um 15\%Punkte durch Pembrolizumab bei Randomisierung von 1000 Patienten. Als sekundäre Endpunkte wurden pCR, EFS und das Gesamtüberleben (OS) unter den Patienten mit PD-L1-positiven TNBC bestimmt.

Ergebnisse Die Beurteilung der pCR-Rate erfolgte an den 602 ersten randomisierten Patienten. Auf eine ähnliche demografische und krankheitsspezifische Ausgangslage zwischen den beiden Gruppen, insbesondere bezüglich des PDL1-Status, wurde geachtet. In der Gruppe mit Pembrolizumabchemotherapie erreichten 64,8\% (95\%-KI: 59,9-69,5) der Patienten die pCR im Vergleich zu nur 51,2\% (95\%KI: 44,1-58,3) in der Gruppe mit Plazebogabe (geschätzter Behandlungsunterschied von 13,6\%; $95 \%$-KI: 5,4-21,8; $p<0,001)$. Gemäß des vorher berechneten Signifikanzwertes von $p=0,003$ für die geplante Interimsanalyse war die pCR damit signifikant höher zugunsten der Pembrolizu- 
mabchemotherapiegruppe. Ein vergleichbarer Unterschied wurde sowohl bei Betrachtung der sekundären Endpunkte in der Gruppe der PD-L1-positiven TNBC als auch bei den Tumoren mit niedrigem PD-L1-Status beobachtet.

Die Progressionsraten nach 18 Monaten nach Kaplan Meier ergaben für 91,3\% der Patienten der Pembrolizumabchemotherapiegruppe und für $85,3 \%$ der Plazebogruppe ein EFS ohne erneutes lokales oder disseminiertes Wiederauftreten des Primärtumors und ohne Sekundärtumor (,,hazard ratio“: 0,63; 95\%-KI: 0,43-0,93). Dort stagnierte das EFS und bildete ein Plateau.

Behandlungsassoziierte Nebenwirkungen vom Grad $\geq 3$ traten in 76,8\% der Patienten der Pembrolizumabchemotherapiegruppe und 72,2\% der Plazebogruppe auf und führten zu 3 bzw. 1 Todesfall in den jeweiligen Gruppen. Zu den nach Pembrolizumab vermehrt auftretenden Nebenwirkungen zählten Exantheme, Infusionsreaktionen und immunitätsbedingte adrenale Insuffizienzen.

Schlussfolgerung der Autoren Pembrolizumab führt zu einer signifikanten Zunahme der pCR bei Patienten mit frühem TNBC. Die erhöhte pCR-Rate tritt in allen Subgruppen und ebenfalls in der Patientengruppe mit einer niedrigen PD-L1-Expression auf.

\section{Kommentar}

Aufgrund der guten Ergebnisse der KEYNOTE-522-Studie bezüglich pCR und OS scheint Pembrolizumab eine sinnvolle Ergänzung zur Chemotherapie als momentan einzig zugelassene neoadjuvante Behandlung zu sein [4, 9]. Dafür spricht ebenfalls, dass aus Metaanalysen bereits bekannt ist, dass eine $\mathrm{pCR}$ insbesondere beim TNBC mit einem verbesserten Gesamtüberleben assoziiert ist $[2,10]$. Auch wegen der Charakterisierung des TNBC als immunogenen Tumor, mit häufig erhöhten $\mathrm{CD}^{+}$-T-Zell-Werten und einer häufig erhöhten PD-L1-Expression, scheinen Immuncheckpointinhibitoren der richtige Ansatzpunkt für eine mehr zielgerichtete Therapie zu sein [4]. Auf Basis der heterogenen Entität des TNBC könnten in Zukunft weitere Studien zur Differenzierung möglicher Unterschiede im Therapieansprechen der einzelnen Subtypen sinnvoll sein [9]. Positiv ist ebenfalls die Kongruenz der Ergebnisse mit der Phase-2-Vorstudie ISPY2, in der bei neoadjuvanter Gabe von Pembrolizumab bei frühen TNBC sogar eine Verbesserung der pCR um 40 Prozentpunkte erreicht werden konnte [3, 11].

Auch bei der Behandlung von metastasierten TNBC mit Pembrolizumab als Monotherapie konnte in der Phase-1Studie KEYNOTE-12 ein Gesamtansprechen von 18,5\% erreicht werden $[4,8,9]$ sowie in der Phase-2-Studie KEYNOTE-086 ebenfalls ähnliche Ergebnisse bezüglich des progressionsfreien Überlebens (PFS) und des OS. In der KEYNOTE-086-Studie trat in der Kohorte vorher schon einmal behandelter Patienten ebenfalls kein signifikanter Unterschied im PFS oder OS in Bezug auf den PD-L1Status auf [4]. Allerdings zeigte sich ein besseres Therapieansprechen, wenn Pembrolizumab in der Erstbehandlung von PD-L1-positiven Tumoren eingesetzt wurde [13].

Der Behandlungsvorteil durch Pembrolizumab, auch bei niedriger PD-L1-Expression, zeigt, dass die fehlende Expression von Biomarkern kein Ausschlusskriterium für eine Therapie oder Studienteilnahme sein muss [6]. Sowohl die KEYNOTE-522- als auch die der KEYNOTE-086-Studiengruppe planen weitere Analysen der Biomarker in ihrem Patientenkollektiv, was vor diesem Hintergrund notwendig und sinnvoll erscheint $[3,12,13]$. Durch retrospektive vergleichende Analysen der Unterschiede und Nebenwirkungen zwischen den Subgruppen könnten evtl. weitere Befunde erhoben werden, die dazu beitragen könnten, evtl. immunvermittelte, irreversible Nebenwirkungen zu vermeiden.

\section{Fazit}

Die neoadjuvante Gabe von Pembrolizumab beim triplenegativen Mammakarzinom (TNBC) scheint Erfolg versprechend $\mathrm{zu}$ sein, und die Nebenwirkungen erscheinen tragbar [12]. Obwohl der Gewinn bezüglich des Langzeitüberlebens noch kritisch bewertet werden muss [3], sind der signifikante Unterschied im EFS und das dabei erreichte Plateau starke Indizien für eine positive Einschätzung hinsichtlich OS und Heilung. Auch die durch Pembrolizumab erreichte hohe pCR-Rate spricht für einen wahrscheinlichen Vorteil im Langzeitüberleben. Wahrscheinlich werden die jetzigen Behandlungsleitlinien beim TNBC geändert werden müssen $[3,6]$.

\section{Friederike Dierks, Elisabeth Pietsch und Jürgen Dunst, Kiel}

Funding Open Access funding provided by Projekt DEAL.

Interessenkonflikt F. Dierks, E. Pietsch und J. Dunst geben an, dass kein Interessenkonflikt besteht.

Open Access Dieser Artikel wird unter der Creative Commons Namensnennung 4.0 International Lizenz veröffentlicht, welche die Nutzung, Vervielfältigung, Bearbeitung, Verbreitung und Wiedergabe in jeglichem Medium und Format erlaubt, sofern Sie den/die ursprünglichen Autor(en) und die Quelle ordnungsgemäß nennen, einen Link zur Creative Commons Lizenz beifügen und angeben, ob Änderungen vorgenommen wurden.

Die in diesem Artikel enthaltenen Bilder und sonstiges Drittmaterial unterliegen ebenfalls der genannten Creative Commons Lizenz, sofern sich aus der Abbildungslegende nichts anderes ergibt. Sofern das betreffende Material nicht unter der genannten Creative Commons Lizenz steht und die betreffende Handlung nicht nach gesetzlichen Vorschrif- 
ten erlaubt ist, ist für die oben aufgeführten Weiterverwendungen des Materials die Einwilligung des jeweiligen Rechteinhabers einzuholen.

Weitere Details zur Lizenz entnehmen Sie bitte der Lizenzinformation auf http://creativecommons.org/licenses/by/4.0/deed.de.

\section{Literatur}

1. Schneeweiss A, Denkert C, Fasching PA et al (2019) Diagnosis and therapy of triple-negative breast cancer (TNBC) - recommendations for daily routine practice. Geburtshilfe Frauenheilkd 79(06):605-617

2. Cortazar P, Zhang L, Untch M et al (2014) Pathological complete response and long-term clinical benefit in breast cancer: The CTNeoBC Pooled Analysis. Lancet 384(9938):164-172

3. Killock D (2020) Moving pembrolizumab forwards. Nat Rev Clin Oncol 17(4):196

4. Katz H, Alsharedi M (2018) Immunotherapy in triple-negative breast cancer. Med Oncol 35(1):1-9

5. Hudis CA, Gianni L (2011) Triple-negative breast cancer: An Unmet Medical Need. The Oncol 16(1):1-11

6. Ileana-Dumbrava E (2020) Boosting immune responses against early breast cancer. Sci Transl Med 12(535): 1

7. Janni W, Arbeitsgemeinschaft Gynäkologische Onkologie e. V. in der Deutschen Gesellschaft für Gynäkologie und Geburtshilfe e. V. und der Deutschen Krebsgesellschaft e. V. (2020) Diagnose und Behandlung von Patienten mit primärem und metastasiertem Mammakarzinom. Guidelines Breast Version 2020.1D. Zuckschwerdt, München, p 1-603

8. Fares J, Kanojia D, Rashidi A et al (2020) Landscape of combination therapy trials in breast cancer brain metastasis. Int J Cancer. https://doi.org/10.1002/ijc.32937

9. Liedtke C (2017) Fortgeschrittenes Triple-negatives Mammakarzinom: Immuntherapien in Prüfung. Dtsch Ärztebl Perspekt Onkol. https://doi.org/10.3238/PersOnko/2017.06.16.03

10. Fasching PA (2019) Meta-analysis on association of pathological complete response with long-term survival outcomes in triple-negative breast cancer. Ann Oncol. https://doi.org/10.1093/annonc/ mdz240.067

11. Nanda R, Liu MC, Yau C et al (2020) Effect of pembrolizumab plus neoadjuvant chemotherapy on pathologic complete response in women with early-stage breast cancer. JAMA Oncol 6(5):1-9

12. Stirrups R (2020) Pembrolizumab for triple-negative breast cancer. Lancet Oncol 21(4):183. https://doi.org/10.1016/S1470-2045(20) 30154-6

13. Adams S, Loi S, Toppmeyer D et al (2019) Pembrolizumab monotherapy for previously untreated, PD-L1-positive, metastatic triplenegative breast cancer. Ann Oncol 30(3):405-411 